\title{
Egypte: Développer l'accès aux soins après avortement
}

Frontiers in Reproductive Health

Follow this and additional works at: https://knowledgecommons.popcouncil.org/departments_sbsr-rh

Part of the International Public Health Commons, Public Health Education and Promotion Commons, and the Women's Health Commons How does access to this work benefit you? Let us know!

\section{Recommended Citation}

"Egypte: Développer l'accès aux soins après avortement," FRONTIERES Résumés de Recherche Opérationnelle. Dakar: Population Council, 2000. 


\section{Egypte Développer l'accès aux Soins \\ Soins \\ Après Après Avortement \\ Avortement}

$N^{\circ} 12$

La formation des prestataires et l'introduction d'un protocole de gestion de cas ont permis d'améliorer les Soins Après Avortement (SAA) dans dix hôpitaux universitaires et gouvernementaux d'Egypte. Des patientes ont témoigné que l'attente était moins longue et qu'elles étaient très satisfaites des services médicaux reçus. Les médecins ont adopté des méthodes de traitement entraînant moins de complications et ont fourni aux patientes plus d'informations sur leur état de santé.

\section{Contexte}

Une étude pilote menée en 1994 dans deux hôpitaux égyptiens a prouvé que le perfectionnement des SAA et la formation des médecins aux techniques d'Aspiration Manuelle Intra-Utérine (AMIU), au contrôle des infections et au counseling a conduit à l'amélioration significative des SAA apportés aux patientes.

L'étude réalisée en 1997 par Egyptian Fertility Care Society, avec l'appui du Population Council, avait pour objectif d'institutionnaliser la mise à jour des SAA et des procédures de counseling dans dix hôpitaux dont sept gouvernementaux et trois universitaires.

Pendant l'intervention de 1997, cinq médecinschefs de chaque hôpital, ont pris part à une formation de cinq jours sur l'AMIU, le contrôle des infections et le counseling sur la

Planification Familiale (PF). Ces médecins ont ensuite dispensé pendant quatre mois une formation continue aux médecins et infirmiers dans dix hôpitaux. Un protocole de gestion, comprenant le traitement médical d'urgence, le contrôle de la douleur et le counseling sur la PF a aussi été introduit dans ces hôpitaux. Des chercheurs ont évalué les résultats de changements observés au niveau de la connaissance et de la pratique ; en effectuant des entretiens avant l'intervention (sur 255 médecins, 311 infirmiers et 508 patientes) et après l'intervention (sur 246 médecins, 263 infirmiers et 497 patientes). Des données ont aussi été collectées dans 1036 dossiers médicaux.

\section{Résultats}

- Après leur formation les médecins ont acquis des connaissances supplémentaires sur les complications à court terme, les impacts négatifs sur la santé et le retour immédiat à la fécondité suite aux SAA. Ils ont presque tous reconnu que les patientes ayant bénéficié des SAA doivent recevoir des informations sur la cause des fausses couches et la nécessité de bien se nourrir, se reposer et d'utiliser une méthode contraceptive durant la période de convalescence.

\section{Soins reçus par les patientes en SAA}

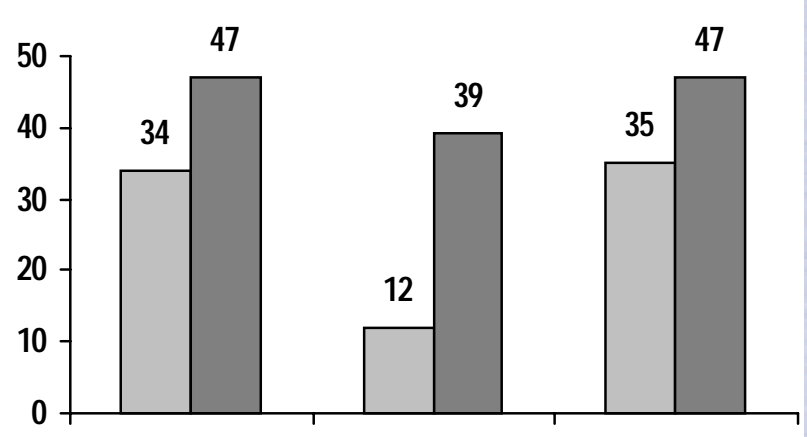

Patientes informées Patientes ayant reçu Patientes ayant reçu sur les problèmes des rendez-vous des informations sur post-opératoires pour un suivi

la PF

$\square$ Avant la formation $\square$ Après la formation 
Aussitôt après cette formation, les médecins ont préféré la méthode AMIU à celle universelle de Dilatation et du Curetage (DC) dans 57 pourcent des cas nécessitant un traitement médical d'urgence. Plus de trois médecins sur quatre ont témoigné qu'ils rencontraient moins de complications avec la méthode AMIU qu'avec la méthode DC. Plus de la moitié ont témoigné que la méthode AMIU était plus efficace et plus facile à utiliser que la méthode DC.

Dans environ trente pourcent des cas, recourir à la méthode AMIU équivaut à passer de l'anesthésie générale à l'anesthésie locale. Suite à cette formation, la proportion de médecins, ayant déclaré que l'application d'analgésie légère et de l'anesthésie locale pouvait réduire l'anxiété des patientes, s'est accrue de manière significative. Toutefois, les techniques de contrôle de la douleur doivent être améliorées : 18 pourcent des patientes n'ont reçu aucun traitement de contrôle de la douleur. Bien que la proportion des patientes ayant témoigné avoir ressenti des douleurs graves après leur interventions ne soit pas accrue de manière considérable, le nombre de patientes ayant témoigné avoir ressenti des douleurs modérées était cinq fois supérieur (de 5 pourcent à 27 pourcent ).

Après intervention, près de trois sur quatre (73 pourcent) des patientes ayant reçu les SAA ont déclaré que les services reçus à l'hôpital étaient excellents comparées à 44 pourcent avant intervention. Comparé aux patientes ayant reçu des soins avant intervention, la majorité des patientes ayant reçues des soins après intervention ont déclaré que l'accueil était amical, l'attente pour les services médicaux a duré moins de 30mn et qu'elles ont été informées de possibles complications, de comment les gérer et faire le suivi.
La proportion des patientes SAA ayant déclaré avoir reçu des informations sur la planification familiale à l'hôpital est passée de 37 pourcent à 47 pourcent. Toutefois, seulement 7 pourcent de ces patientes ont reçu une méthode contraceptive avant leur sortie d'hôpital.

\section{Implications pour les programmes}

$\checkmark$ La formation et les protocoles de SAA, comprenant les procédures de contrôle de la douleur et des infections devraient être uniformisés au niveau des hôpitaux ainsi que des écoles de santé et des facultés de médecine. Les hôpitaux gouvernementaux et universitaires doivent considérer l'AMIU comme élément standard dans leur budget et s'assurer de la fourniture adéquate des la médication relative à la douleur.

\section{Quelques aspects des SAA doivent être} améliorés : contrôle de la douleur, informations données aux patientes et services de counseling sur la PF. Les infirmiers doivent jouer un rôle plus important dans le bien-être, le counseling et l'information des patientes des SAA. Des guides simples à utiliser sur les instruments de la méthode AMIU et le counseling sur la PF doivent être élaborés.

\section{Utilisation}

- Le projet «Mère en bonne santé/Enfant en bonne santé» du Ministère de la Santé et de la Population forme les agents devant utiliser la méthode AMIU et les autres éléments des SAA, notamment le contrôle de la douleur en rapport avec les services de planification familiale. Dix nouveaux hôpitaux ont introduit une méthode améliorée des SAA grâce au projet Mère en Bonne santé/Enfant en Bonne santé - 5 à Aswan, 2 à Luxor et 3 au Qena Sud. L'introduction de cette méthode dans de nouveaux sites à Fayoum et Bani Sewef est en cours.

Mai 2000

Nawar, Laila et al., « Augmenter proportionnellement les Soins Après Avortement Améliorés en Egypte : Introduction dans les Hôpitaux Universitaires et du Ministère de la Santé et de la Population ». Pour plus d'information, contacter : le Population Council,

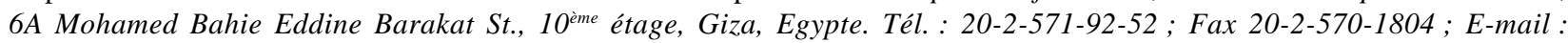
frontiers@pccairo.org.

Ce projet a été entrepris avec l'appui de l'AGENCE AMERICAINE POUR LE DEVELOPPEMENT INTERNATIONAL (USAID) sous le Numéro de Contrat DPE-C-00-90-0002-10 et DPE-3030-Q-00-0023-00.

(1) Population Cound 